\title{
DYNAMIC SELECTION OF CLUSTER HEAD IN IN-NETWORKS FOR ENERGY MANAGEMENT
}

\author{
K.Suriyakala ${ }^{1}$, D.Anandhavalli ${ }^{2}$, C.Suganya ${ }^{3}$ \\ ${ }^{1,3}$ PG Student (Network Engineering), ${ }^{2}$ Assistant Professor-III, Dept of IT, Velammal College of Engineering and \\ Technology, Tamil Nadu, India
}

\begin{abstract}
In this project, we presented Multipath Region Routing (MRR) protocol for energy conservation in Wireless Sensor Networks (WSNs). Large scale dense WSNs are used in different types of applications for accurate monitoring. Energy conservation is an important issue in WSNs. In order to save energy, Multipath Region Routing protocol is used which provides balance in energy consumption and sustains the network life-span. By using this method, we can reduce the number of energy dissipation because the cluster head will collect data directly from other nodes. Hence, the energy can be preserved and network life time is extended to reasonable time.
\end{abstract}

Keywords: Clustering; Wireless Sensor Networks; Security; Multipath Region Routing;

\section{INTRODUCTION}

A Wireless Sensor Networks (WSN) comprises of spatially distributed small sensing nodes which are autonomous and are called 'Sensor nodes'. They used to monitor the physical or environmental conditions such as pressure, temperature, vibration, sound, motion or pollutions in different locations [1, 2]. WSNs are used in numerous applications such as Industrial and Commercial Uses, Smart Home or Smart Office appliances and their security, critical infrastructure systems, Military Surveillance and Troop Support, communications and Traffic Management and Monitoring which can be used to make our life easy. [3-5]. Nodes of WSNs are more energy constrained and energy consumption is a key issue in such type of networks. This energy depends on the amount of gathered data. So, the communication is a critical task by considering energy.

For that reason, we designed some protocols and algorithms to reduce the energy consumption in WSNs. [6-9]. Generally, the WSNs produce data as prominent which needs to be routed and are called data-driven networks. WSNs are work as a gateway between monitoring center for sink nodes. From the above process, we can know that the WSNs play a vital role in data gathering procedure. In order to achieve the optimization in routing task, we can utilize the available processing capacity which is provided by the intermediate sensor nodes along with the routing paths. This process is known as innetwork data aggregation or data centric routing. By using limited resources, we can get efficient and effective data gathering and local decisions are made by the sensor nodes which are assembled with smartly report data. [10-13]. Data aggregation is an effective technique that can be used to save energy in WSNs.
The sensor nodes collected raw data that contains some inherent redundancy and over all communication cost is reduced by eliminating such redundancies. So, we can forward only some aggregated information. While sending minimum amount of data, we can save the energy in WSNs and the network life-time is extended to reasonable times. The data centric routings plays an important role in WSNs because it is used as a key technology. Here, the terms data aggregation and information fusion are used as synonyms and functions of information fusion can be classified into two types, (i) to increase data accuracy by using advantage of data redundancy. (ii) To save energy by reducing communication load. [14].

Generally, the nodes failures and interruptions in communication are present in a network and these are the major task for routing algorithm which is used to provide guarantee to the delivery of sensed data in WSNs. When data aggregation is performed, the node failures and interruptions becomes more critical task. The aggregated data packet contains information of several resources and if any one of the packets is lost, a significant amount of information will also be lost. In WSNs, routing protocols with data aggregation has some features such as: it has reduced number of messages that is used to construct a routing tree; maximum number of overlapping routes, rate of data aggregation is high and authentic data transmission is possible. To overcome these issues, we suggest Multipath Region Routing protocol for WSNs. Our proposed protocol contains a Cluster Head $(\mathrm{CH})$ in which all the cluster nodes send their data to the $\mathrm{CH}$ and it transfers the collected data to the Base Station. 


\section{EXISTING SYSTEM}

Generally, most of the researches have been conducted on data aggregation techniques, contains a flat sensor networks. For instance, consider a tree based topology that creates a simple parent and child relationship and this simple topology is only utilized by the protocol which is not critical. In this approach, transmission delays are increased and it does not provide any assurance to high aggregation rates. In our paper, we consider the dynamic sensor network rather than the flat sensor networks. Some other researchers have been done to increase the aggregation efficiency in terms of target tracking in hierarchical networks. In order to increase the aggregation efficiency, we use the concept of clustering in which the data aggregation is controlled by hierarchy. $[15,16]$. There are two types of clustering mechanisms are used: one is static clustering and other is dynamic clustering.

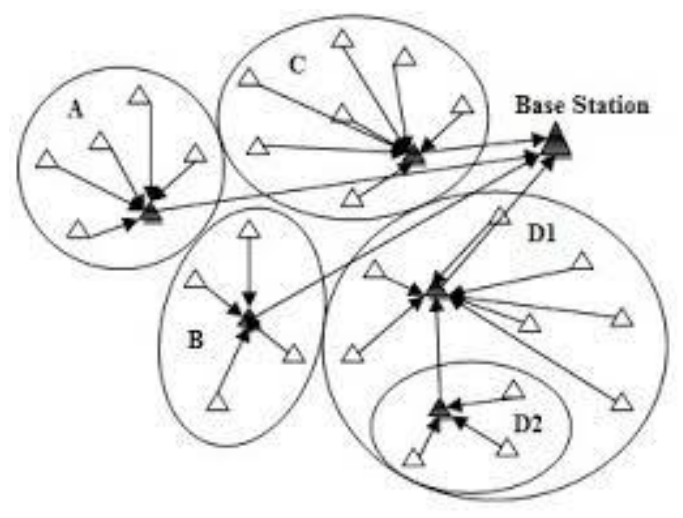

Fig -1: Group Formation Using Cluster Method

In static clustering, the nodes are statistically clustered into many clusters. Moreover, a cluster consists of general cluster nodes and a cluster head and this cluster head gathered data directly from the general cluster nodes. Then, the cluster head must aggregate several data packets which are sent towards the sink nodes. While data aggregation takes place in static clustering it utilizes the pre-elected cluster heads. By using this aggregation, we can attain low over-heads because the data packets are transmitted easily in a rapid manner. Every time in a network, great overheads are caused by static clusters periodically and by reducing such overheads, each node must have a flood control packets. [18]

When two or more clusters will sense the destination at the same time, the aggregation efficiency can be reduced. To obtain high efficiency in data aggregation at static clustering, all the sensing arrangements have been done in a single node. This method uses multi hop clusters and this is the major drawback of static clustering. This will reduce the number of clusters and increase the cluster size. Here, the average number clusters which sense the target at the same time will be reduced. [17,19]. But, additional overhead problem takes place in this case because data aggregation towards the cluster head may have more than one hop.

In dynamic clustering, clusters are created in the case of sensing occurs to the neighborhood nodes. After event sensing, a cluster head will be chosen that can sense the destination among the other nodes and at the same time, the other nodes also sense the destination will turn to general nodes which are placed in a single hop location of cluster in the network. The cluster head is acted as a Leader node and it collects the information from all other nodes. Then it will send the information to the sink node. Here, only essential nodes can take part in the event of data aggregation. So we can preserve the energy of other nodes and this is the main advantage of this technique. In static clustering, cluster head can disseminate the control packets and by the way it can reduce the control packet overhead. [20].

In case of dynamic clustering, all the data aggregation can be attained in neighborhood sensing and the data does not travel through various hops that are to be aggregated. By considering this case, data aggregation rate is enormously high in dynamic clustering networks and this method has some other drawbacks. In the event of electing the cluster head, the delay is increased because at first the sensing event takes place before transmitting the data. Therefore, data aggregation will occurs only after the cluster election. If the velocity of target is relatively very high, then only the dynamic clusters are created in a rapid manner.[21,22]. To overcome these drawbacks, we introduce Multipath Region Routing protocol.

\section{SOURCES OF ENERGY WASTAGE}

As, in this section we have discussed about the four major sources of energy wastage. At first, consider the case of collision. If a transmitted packet is corrupted due to collision, then it will be discarded. In this case, retransmission is needed to transmit the same packet and then energy consumption and latency are increased. Second source of energy wastage is overhearing which means a node that catches the packets allocated for other nodes. Control packet overhead is the third

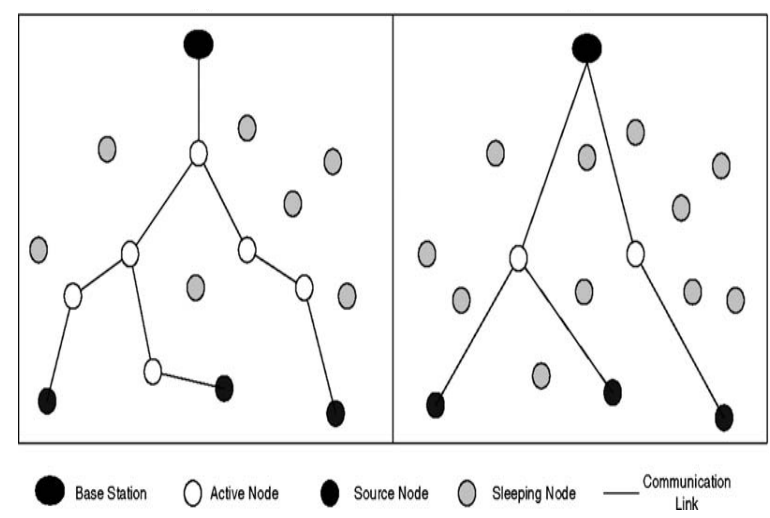

Fig -2: Sources of Energy Wastage 
Case that takes more energy while transmission and reception of packets occur and these packets may contain unwanted information's. Finally, we talk about the major source of energy wastage is idle listening which involves to listen the possible unsent traffic. This type of energy wastage happens in many applications of sensor networks and typically the nodes are in idle state because there is nothing is sensed in this network.

\section{PROPOSED SYSTEM}

In our paper, we proposed Multipath Region Routing (MRR) protocol for energy conservation in WSNs. Multipath Region Routing protocol is one of the methods for transmitting packets to their destination in an efficient manner. When the recent path cannot able to transmit the available packets, it chooses the alternate path for packet transmission and a group of packets are delivered to the destination without any delay. This protocol reduces the delay so that the network performance is increased. Hence, the network provides efficient packet transmission with scalable and robust communication.

It also reduces the network traffic by the way this protocol increases the communication living time. In order to enhance the packet delivery ratio, we required reliable communication with any unwanted interruption among the nodes. Multipath Region Routing protocol attains low latency and high succession ratio. Thus, MRR protocol reduces the network cost when compared to other protocols.

\section{ALGORITHM FOR MULTIPATH REGION ROUTING PROTOCOL}

Initialize the Source Node $S_{i x}$

Formulate the Routing Process $R_{i x}$

Formulate the Routing Process $D_{i x}$

$S_{i x<-} R_{i x}+D_{i x} / /$ Packets Transfer Source to Destination travel to Several Paths using Routing process

Path Selection Process $P_{\text {sel }}$

Choose Alternate path $P_{\text {Apath }}$

For $\left(N_{i x=0 ;} N_{i x<22 ;} N_{i x++)}\right.$

If( $\left.N_{i x}=0\right)$;

$N_{i x}=$ Packet Dropping (or)Unknown Packet

Else

$N_{i x}=P_{\text {Apath }}$

Calculate the packet Dropping Using Packet Id

Calculate the Overall Network performance

\subsection{Description}

Generally, nodes are deployed in a network and in our project nodes are arranged in a dynamic manner. When the packet transmission is initiated, it prefers several paths to deliver the packets to the particular destinations. Here, we have 10 paths for packet transmission, if the packet loss is increased by using any one of them; it chooses the alternate one. Then, the packet dropping is also available in the current path; it chooses the alternative for transmission. The above process is repeated to achieve higher throughput. Finally, the packet dropping ratio and overall network performance is calculated.

\section{CONCLUSION AND FUTURE WORK}

Thus, we presented a novel of Cluster Formation Using Zone Leader Election (CFZLE) algorithm for energy conservation in WSNs and our proposed method has more beneficial features when compared to traditional cluster based approaches. During transmission, the consumption of energy can be saved by splitting the nodes into clusters and the loss of energy can be detected by calculating the packet performance of nodes at each zones. Then, overall network performance is calculated after the completion of packet transmission in all zones in the network. Finally, compare the overall performance of proposed and the traditional method of packet transmission. Our future work will concentrate on the implementation of CFZLE algorithm using NS-2 by distinguished module so only we can tested the output more accurately. Then, we will measure energy consumption of the nodes. By the way, we can evaluate the network lifetime.

\section{EXPERIMENTAL RESULT}

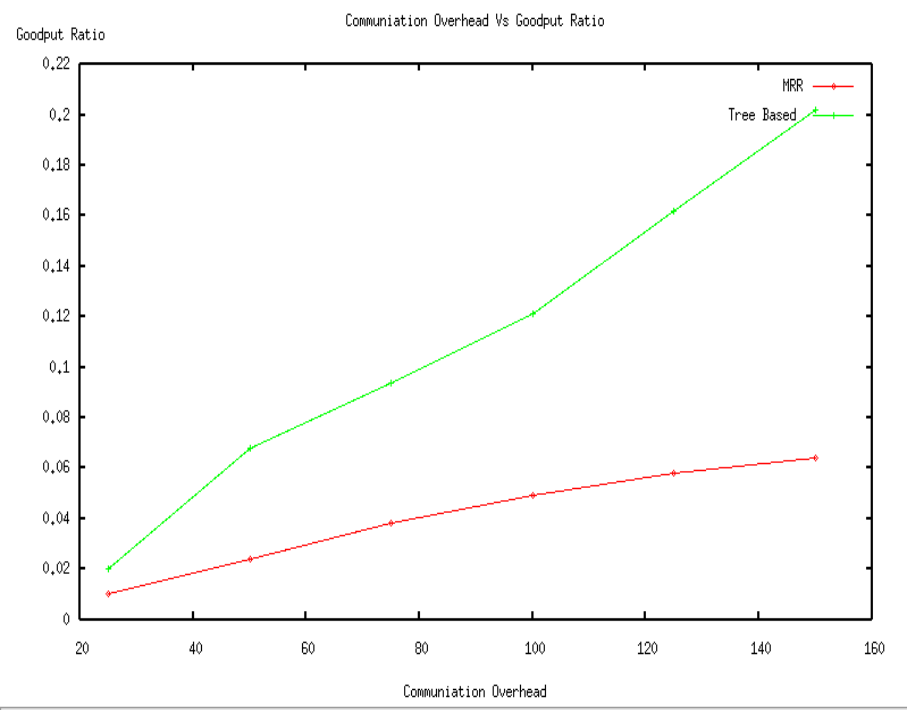

Fig -3: Performance Compare between Communication Overhead Vs Good put 


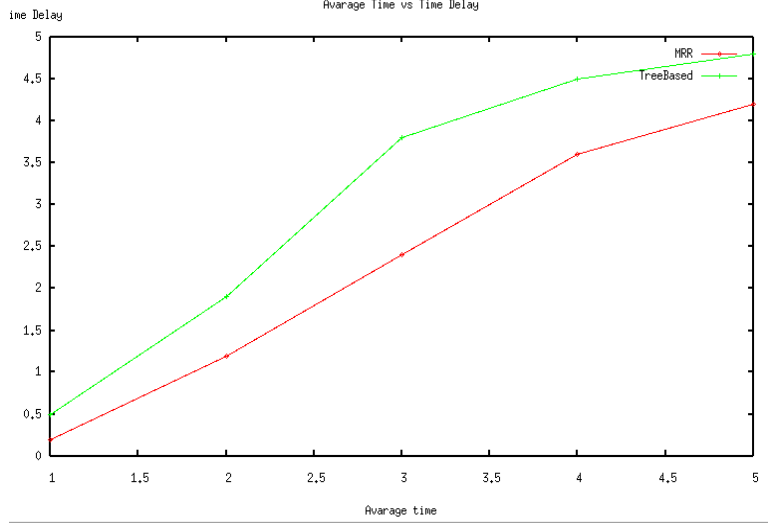

Fig -4: Performance Compare between Average Time Vs Time Delay

\section{REFERENCES}

[1]. Intel Research Berkeley, "Collaborating to Change the Wold", Ver. 1, 2004

(http:// www.intel.com/research/print/berkeley_collab.pdf)

[2]. E. Blomqvist, H. N. Koivo, "Security In Sensor Networks - A Case Study", 12th Med. Conf. On Control and Automation MED’04 (IEEE), Kusadasi, Turkey, 2004.

[3]. J. Rabaey, E. Arens, C. Federspiel, A. Gadgil, D. Messerschmitt, W. Nazaroff, K. Pister, S. Oren, P. Varaiya, White Paper: "Smart Energy Distribution and Consumption: Information Technology As An Enabling Force", (www.citris.berkeley.edu/SmartEnergy/SmartEnergy.html)

[4]. M. B. Srivastava, R. R. Muntz, and M. Potkonjak, "Smart kindergarten: sensorbased wireless networks for smart developmental problem-solving environments". In Mobile Computing and Networking, pp. 132-138, 2001.

[5]. L. Schwiebert, S. K. S. Gupta, and J. Weinmann, "Research challenges in wireless networks of biomedical sensors". In Mobile Computing and Networking, pp. 151-165, 2001.

[6]. I. F. Akyildiz, W. Su, Y. Sankarasubramaniam, and E. Cayirci, "Wireless sensor networks: a survey", Computer Networks 38, Elsevier, pp. 393-422, 2002.

[7]. G. T. Sibley, M. H. Rahini, G.S. Sukhatme, " Robomote: A Tiny Mobile Robot Platform for Large-Scale Ad-hoc Sensor Networks", In Proc. of the Intl. Conf. on Robotics and Automation Washington DC, Sep 2002.

[8]. K. Dantu, M. Rahimi, H. Shah, S. Babel, A. Dhariwal, and G. Sukhatme, "Robomote: Enabling Mobility In Sensor Networks", ACM Journal, Vol. 1, No. 1, 122004.

[9]. D. J. Baker and A. Epheremides, "The Architectural Organization of a Moblie Radio Network via a Distributed Algorithm," IEEE Transactions on Communications, vol. Com-29, no. 11, November 1981.

[10]. P. Tsigas, "Project on Moblie Ad Hoc Networking and Clustering for the Course EDA390 Computer Communcation and Distributed Systems," Manual for University Course.
[11]. A. Amis, R. Prakash, T. Vuong, and D. Huynh, "MaxMin D-Cluster Formation in Wireless Ad Hoc Networks," IEEE INFOCOM, March 2000.

[12]. C.E.Nishimura and D.M.Conlon, "IUSS dual use: Monitoring of whales and earthquakes using SOSUS," Mar. Technol. Soc. J., vol. 27, no. 4, 1994.

[13]. A. Mainwaring et al., "Wireless Sensor Networks for Habitat Monitoring," Proceedings of the 1st ACM International Workshop on WSN, 2002.

[14]. C.Y.Chong, S.Mori, and K.C.Chang, "Distributed multitarget multisensor tracking," in Multitarget Multisensor Tracking:Advanced Applications, 1990.

[15]. C. Intanagonwiwat et al., "Directed Diffusion for Wireless Sensor Networking," IEEE/ACM Transaction on Networking, vol. 11, no. 1, Feb. 2003.

[16]. Xue, Q.; Ganz, A. Maximizing Sensor Network Lifetime: Analysis and Design Guides. In Proceedings of MILCOM, October 2004.

[17]. Chan, H.; Perrig, A. ACE: An Emergent Algorithm for Highly Uniform Cluster Formation. In Proceedings of the First European Workshop on Sensor Networks (EWSN), 2004.

[18]. Ye, M.; Li, C.F.; Chen, G.; Wu, J. EECS: An Energy Efficient Clustering Scheme in Wireless Sensor Networks. Int. J. Ad Hoc Sensor. Network. 2007, 3, 99-119.

[19]. J.M. McCune. Adaptability in sensor networks. Undergraduate Thesis in Computer Engineering, University of Virginia, April 2003.

[20]. I. Texas Instruments, "MSP430x13x, MSP430x14x Mixedm Signal Microcontroller. Datasheet, " 2001.

[21]. W. Heinzelman, A. Chandrakasan, and H. Balakrishnan, "An Application-Specific Protocol Architecture for," IEEE Transactions on Wireless Communications, vol. 1, 2002. [22]. O. Younis and S. Fahmy, "HEED: A Hybrid, EnergyEfficient, Distributed Clustering Approach for Ad Hoc Sensor Networks," IEEE Trans. Mobile Computing, vol. 3, no. 4, pp. 660-669, Oct.-Dec. 2004. 